\title{
Dobutamine-sparing strategy in managing patients with impaired ejection fraction undergoing coronary artery bypass grafting: less is more?
}

\author{
Xiao-Ming Lin ${ }^{1 \#}$, Ming-Hao Luo ${ }^{2 \#}$, Jing-Chao Luo ${ }^{3}$, Kanhua Yin ${ }^{4}$, Ying Zhang ${ }^{3}$, Xiao-Mei Yang ${ }^{3}$, \\ Zhe Luo ${ }^{1,3}$, Guo-Wei Tu ${ }^{3}$ \\ ${ }^{1}$ Department of Critical Care Medicine, Xiamen Branch, Zhongshan Hospital, Fudan University, Xiamen, China; ${ }^{2}$ Shanghai Medical College, Fudan \\ University, Shanghai, China; ${ }^{3}$ Department of Critical Care Medicine, Zhongshan Hospital, Fudan University, Shanghai, China; ${ }^{4}$ Department of \\ Surgery, Massachusetts General Hospital, Harvard Medical School, Boston, MA, USA \\ "These authors contributed equally to this work. \\ Correspondence to: Zhe Luo. Department of Critical Care Medicine, Xiamen Branch, Zhongshan hospital, Fudan University, Xiamen, China. \\ Email: luo.zhe@zs-hospital.sh.cn; Guo-Wei Tu. Department of Critical Care Medicine, Zhongshan Hospital, Fudan University, Shanghai, China. \\ Email: tu.guowei@zs-hospital.sh.cn.
}

Submitted Mar 03, 2021. Accepted for publication May 14, 2021.

doi: $10.21037 /$ jtd-21-363

View this article at: http://dx.doi.org/10.21037/jtd-21-363

In many centers worldwide, inotropic therapy has been used routinely in managing patients undergoing cardiovascular surgery and is considered a standard of care. However, few high-quality randomized controlled trials (RCTs) have verified its efficacy, and there is no consensus on how inotropes should be used in cardiac surgery patients $(1,2)$. Recently, an RCT has shown that the 30-day mortality and major cardiovascular complication risks were similar between the dobutamine-sparing and the dobutamine-toall strategy groups in patients with normal left ventricular ejection fraction (LVEF) (i.e., LVEF $>50 \%$ ) who underwent coronary artery bypass grafting (CABG) (3). This study showed that in patients with normal LVEF, the dobutaminesparing strategy was not inferior to the dobutamine-toall strategy in terms of short-term clinical outcomes and highlighted that the pros of dobutamines (quick myocardial contractility recovery) may not outweigh its cons (increase in myocardial oxygen demand) in patients undergoing CABG.

Motivated by the result, we would like to explore whether their conclusions can be extended to patients with impaired LVEF in our own patients. Population-based studies have shown that as high as $35 \%$ of patients undergoing CABG had LVEF less than $40 \%$ (4) while no RCTs has studied the effects of dobutamine in this particular group of patients. In our center (Zhongshan Hospital, Fudan University), we follow a strategy of using inotropes in CABG patients as little as possible: dobutamine is not routinely given to CABG patients but only reserved for those who are complicated with low cardiac output syndrome. In addition, $\beta$-blocker is routinely used from day one after cardiac surgery when the patient weans off the vasopressor.

From January 2018 to December 2020, 1,242 patients underwent either on-pump or off-pump CABG at out center. Due to surgeons' preference, off-pump CABG is the first-line procedure in our center, and on-pump CABG is usually reserved for those with relatively higher surgical risks. To avoid the potential confounding from the surgical procedure, we included 90 consecutive patients who underwent on-pump CABG and dichotomized them into two groups based on their preoperative LVEF ( $>50 \%$ vs. $\leq 50 \% ; 62$ vs. 28 patients). The demographics, preoperative data, and in-hospital outcomes of the two groups were summarized and compared in Table 1. The median EuroSCORE II was 3.5 (IQR, 2-4.8) and 4 (IQR, $2-6)$ in the LVEF $>50 \%$ group and LVEF $\leq 50 \%$ group, respectively, suggesting that these patients had a higher risk than those in Franco et al.'s study (median EuroSCORE II $=2$ in both groups).

On admission to postoperative ICU, about half of patients in both groups were given dobutamine. The use of dobutamine was decreased quickly in both groups 
Table 1 Demographic data, preoperative characteristics and outcomes according to the study group: LVEF $>50 \%(\mathrm{n}=62)$ and LVEF $\leq 50 \%$ ( $\mathrm{n}=28$ )

\begin{tabular}{|c|c|c|c|c|}
\hline Variable & All patients $(n=90)$ & LVEF $>50 \%(n=62)$ & LVEF $\leq 50 \%(n=28)$ & $P$ value \\
\hline Age [years], median [IQR] & 66 [58-72] & $66[62-71]$ & $63[56-72]$ & $0.28^{a}$ \\
\hline Sex (male), n (\%) & $69(76.6)$ & $45(72.5)$ & $24(85.7)$ & $0.32^{\mathrm{a}}$ \\
\hline EuroSCORE II, median [IQR] & $3[2-5]$ & $3[2-4.8]$ & $4[2-6]$ & $0.21^{a}$ \\
\hline Number of graft, median [IQR] & $3[3-3]$ & $3[3-3]$ & $3[3-3]$ & $0.58^{a}$ \\
\hline \multicolumn{5}{|l|}{ Outcomes } \\
\hline Death, n (\%) & $2(2.2)$ & $2(3.2)$ & 0 & $1.00^{\mathrm{c}}$ \\
\hline Arrhythmias, n (\%) & $25(27.7)$ & $18(29.0)$ & $7(25.0)$ & $0.69^{b}$ \\
\hline Stroke, n (\%) & $3(3.3)$ & $3(4.8)$ & 0 & $0.55^{c}$ \\
\hline Renal replacement therapy, n (\%) & $1(1.1)$ & 0 & $1(3.5)$ & $0.31^{\mathrm{c}}$ \\
\hline Septic shock, n (\%) & $2(2.2)$ & $2(3.2)$ & 0 & $1.00^{\mathrm{C}}$ \\
\hline Mechanical ventilation >48 h, n (\%) & $11(12.2)$ & $7(11.2)$ & $4(14.2)$ & $0.73^{\mathrm{c}}$ \\
\hline Length of ICU stay [days], median [IQR] & $3[1-4]$ & $3[1-4]$ & $3[1.75-4.25]$ & $0.70^{\mathrm{a}}$ \\
\hline Length of hospital stay [days], median [IQR] & $14.5[12-18]$ & 14 [12-18] & 15 [12-18] & $0.56^{\mathrm{a}}$ \\
\hline ICU readmission, $\mathrm{n}(\%)$ & $3(3.3)$ & $2(3.2)$ & $1(3.5)$ & $1.00^{\mathrm{c}}$ \\
\hline
\end{tabular}

${ }^{a}$, Mann-Whitney test; ${ }^{b}$, Pearson's Chi-square test; ${ }^{c}$, Fisher's exact test. LVEF, left ventricular ejection fraction; ICU, intensive care unit; IQR, interquartile range.

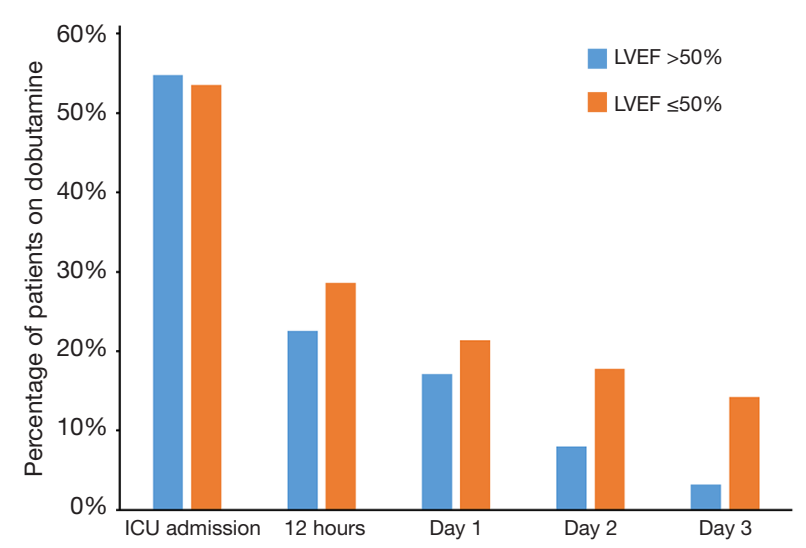

Figure 1 Use of dobutamine in the first 3 days after surgery according to the study group: LVEF $>50 \%(n=62)$ and LVEF $\leq 50 \%(n=28)$. LVEF, left ventricular ejection fraction. with time (first 12 hours: $22.6 \%$ vs. $28.6 \%, \mathrm{P}=0.54$ ) and continued tapering in the next three days (Day 1: 17.1\% vs. $21.4 \%, \mathrm{P}=0.68$; Day 2: $8.0 \%$ vs. $17.8 \%, \mathrm{P}=0.17$; Day 3: $3.2 \%$ vs. $14.2 \%, \mathrm{P}=0.05$; Figure 1 ). Norepinephrine was administered to most CABG patients on ICU admission. Similarly, the use of norepinephrine was also decreased in both groups with time (Day 1: $30.6 \%$ vs. $53.5 \%, \mathrm{P}=0.04$; Day 2: $17.1 \%$ vs. $17.8 \%, \mathrm{P}=0.99$; Day $34.8 \%$ vs. $17.8 \%$, $\mathrm{P}=0.04$; Figure 2).

There were no statistically significant differences between two groups in postoperative arrhythmia $(29.0 \% \mathrm{vs}$. $25.0 \%, \mathrm{P}=0.69)$, cardiogenic shock $(3.2 \%$ vs. $0 \%, \mathrm{P}=1.00)$, stroke $(4.8 \%$ vs. $0 \%, \mathrm{P}=0.55)$, and in-hospital mortality ( $3.2 \%$ vs. $0, \mathrm{P}=1.00)$. The ICU-stay [3 (1.00-4.00) vs. 3 (1.75-4.25) days, $\mathrm{P}=0.70]$, hospital-stay [14 (12.00-18.00) 


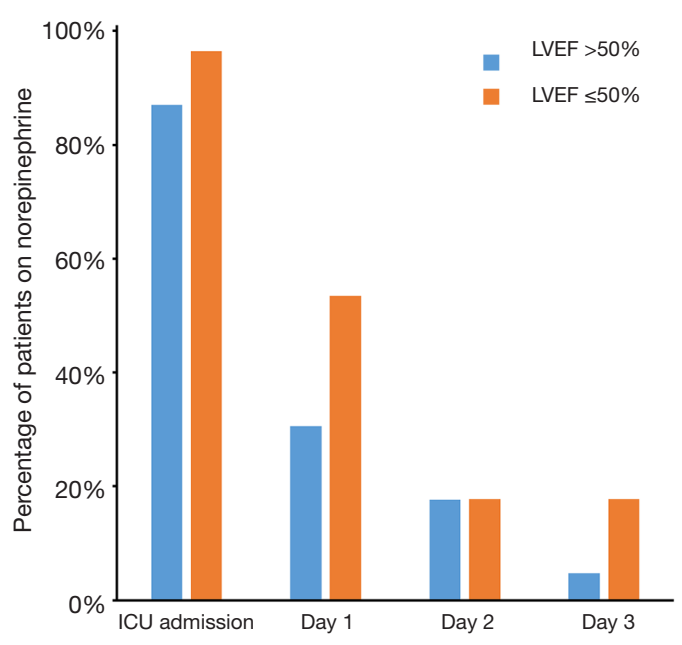

Figure 2 Use of norepinephrine in the first 3 days after surgery according to the study group: LVEF $>50 \%(n=62)$ and LVEF $\leq 50 \%(\mathrm{n}=28)$. LVEF, left ventricular ejection fraction.

vs. 15 (12.00-18.75) days, $\mathrm{P}=0.56$ ], and ICU readmission ( $3.2 \%$ vs. $3.5 \%, \mathrm{P}=1.00)$ were also similar between two groups (Table 1).

Based on our data, a dobutamine-sparing strategy seems feasible and safe even in patients with impaired LVEF. However, dobutamine-sparing strategy was more radical in our center as fewer patients received dobutamine $12 \mathrm{~h}$, 1,2 and 3 days after operation. Interestingly, similar outcomes were found in our patients even with impaired heart function, suggesting "less is more" when it comes to dobutamine use in cardiac surgery.

We acknowledge limitations. First, the dosages of catecholamine and norepinephrine were not evaluated in detail. Different dosages of dobutamine or norepinephrine may contribute differently to myocardial function recovery and the ultimate clinical outcomes. Second, although we adopted a strategy of using inotropes as little as possible, the actual decision of giving (or not giving) dobutamine still largely depended on the individual intensivist's judgment. Heterogeneity may exist in the indication and timing of dobutamine usage. High-quality evidence is still needed to further elucidate the benefits (or harms) of inotropic agents in managing patients undergoing $\mathrm{CABG}$.

\section{Acknowledgments}

Funding: This article was supported by grants from the Clinical Research Funds of Zhongshan Hospital (2020ZSLC38 and 2020ZSLC27) and Smart Medical Care of Zhongshan Hospital (2020ZHZS01).

\section{Footnote}

Provenance and Peer Review: This article was a standard submission to the journal. The article has undergone external peer review.

Peer Review File: Available at http://dx.doi.org/10.21037/jtd21-363

Conflicts of Interest: All authors have completed the ICMJE uniform disclosure form (available at http://dx.doi. org/10.21037/jtd-21-363). Clinical Research Funds of Zhongshan Hospital were granted to Dr. ZL. Funding of Smart Medical Care of Zhongshan Hospital was granted to Dr. ZL. Clinical Research Funds of Zhongshan Hospital (No.2020ZSLC38) were granted to Dr. GWT. The other authors have no conflicts of interest to declare.

Ethical Statement: The authors are accountable for all aspects of the work in ensuring that questions related to the accuracy or integrity of any part of the work are appropriately investigated and resolved.

Open Access Statement: This is an Open Access article distributed in accordance with the Creative Commons Attribution-NonCommercial-NoDerivs 4.0 International License (CC BY-NC-ND 4.0), which permits the noncommercial replication and distribution of the article with the strict proviso that no changes or edits are made and the original work is properly cited (including links to both the formal publication through the relevant DOI and the license). See: https://creativecommons.org/licenses/by-nc-nd/4.0/.

\section{References}

1. Mauriat P, Bojan M, Soulie S, et al. Impact of the perioperative inotropic support in grown-up congenital heart patients undergoing cardiac surgery: a propensity score adjusted analysis. Ann Intensive Care 2020;10:91.

2. Franco RA, de Almeida JP, Landoni G, et al. Dobutaminesparing versus dobutamine-to-all strategy in cardiac surgery: a randomized noninferiority trial. Ann Intensive Care 2021;11:15. 
3. Scheeren TWL, Bakker J, Kaufmann T, et al. Current use of inotropes in circulatory shock. Ann Intensive Care 2021;11:21.

Cite this article as: Lin XM, Luo MH, Luo JC, Yin K, Zhang Y, Yang XM, Luo Z, Tu GW. Dobutamine-sparing strategy in managing patients with impaired ejection fraction undergoing coronary artery bypass grafting: less is more? J Thorac Dis 2021;13(6):3923-3926. doi: 10.21037/jtd-21-363
4. Topkara VK, Cheema FH, Kesavaramanujam S, et al. Coronary artery bypass grafting in patients with low ejection fraction. Circulation 2005;112:I344-50. 